\title{
UNIQUENESS FOR SECOND ORDER LINEAR PARABOLIC OPERATORS
}

\author{
RUSSELL D. RUPP, JR.
}

I. Introduction. A uniqueness theorem for the second order linear parabolic boundary value problem can be given under fairly unrerestrictive conditions with respect to the coefficients, boundary values, and domain. However, care must be taken to avoid counterexamples such as the following: let $L(u)=x u_{x x}-u_{t}=0$ be defined on $D=\{(x, t): 1<x<2,0<t<\infty\}$. Then if $\operatorname{cl}(D)=$ closure of $D$, consider the boundary conditions $u(x, t)=0$ on $\{t=0\} \cap \operatorname{cl}(D)$ and $\partial u / \partial v(x, t)$ $=0$ on $\{x=1,2\} \cap \mathrm{cl}(D)$ where $v(x, t)=(-1,-t)$ on $\{x=1\} \cap \mathrm{cl}(D)$ and $v(x, t)=(2, t)$ on $\{x=2\} \cap \operatorname{cl}(D) . L(u)$ is uniformly parabolic, but $u(x, t)=e^{-x / t}$ is a nontrivial solution.

The above example contradicts Theorem 8, p. 176 of [2] since that theorem does not exclude derivatives in directions with negative time components. In the following section, we formulate a uniqueness theorem stronger than those stated in [1] and which avoids situations like the above by assuming conditions on the boundary or boundary operator which prevent positive maxima (or negative minima) in the case of homogeneous boundary data.

II. Results. By a domain $D$, we mean an open set in $R^{n+1}$ whose points are denoted by $(x, t)=\left(x_{1}, \cdots, x_{n}, t\right) . \operatorname{cl}(D)$ denotes the closure of a set $D$, int $(D)$ its interior, and $\partial D$ its boundary. For our purposes, $D$ lies above the hyperplane $t=0$ and $D_{T}=D \cap\{0<t<T\}$ is bounded for each $T>0$. Furthermore, we suppose that $B=\operatorname{cl}(D)$ $\cap\{t=0\} \neq \varnothing$ and that any point $P \in D$ can be connected to some point $Q \in B$ by a curve in $D$ with nonincreasing $t$-coordinate. Let $S=\partial D \cap\{0<t<\infty\}$ and $S_{T}=\partial D \cap\{0<t \leqq T\}$ for each $T>0$. Boldface letters like $v=\left(\nu_{1}, \cdots, \nu_{n+1}\right)$ denote vectors in $R^{n+1}$. A point $(x, t) \in \partial D$ and a direction $v$ are said to satisfy the (inside) sphere property if there is a ball $K$ with center $(\bar{x}, \bar{t}), \bar{x} \neq x$ and $K \cap \partial D$ $=(x, t)$ while int $(K) \subseteq D$ and starting from $(x, t),-v$ points into $K$. In particular, the sphere property is satisfied at a point $(x, t)$ with respect to the appropriate directions if $\partial D$ in a neighborhood of $(x, t)$ is a $C^{2}$ manifold whose tangent hyperplane at $(x, t)$ is not of the form $t=$ constant. The analogous statement is not true for $C^{1}$ manifolds. All functions considered will be real-valued. Let $L(u)$ be the operator

Received by the editors November 4, 1968. 
on $D$ defined by

$$
\begin{aligned}
L(u)= & \sum_{i, j=1}^{n} a_{i j}(x, t) u_{x_{i} x_{j}}(x, t)+\sum_{i=1}^{n} b_{i}(x, t) u_{x_{i}}(x, t) \\
& +c(x, t) u(x, t)-u_{t}(x, t)
\end{aligned}
$$

where $a_{i i}$ and $b_{i}$ are bounded. Furthermore assume $L(u)$ is uniformly parabolic on $D$, i.e.

$$
\sum_{i, j=1}^{n} a_{i j}(x, t) \xi_{i} \xi_{j} \geqq \mu>0
$$

for all $(x, t) \in D$ and for all $\xi=\left(\xi_{1}, \cdots, \xi_{n}\right) \in R^{n}$ with $|\xi|^{2}=\xi_{1}^{2}+\cdots$ $+\xi_{n}^{2}=1$. We shall consider boundary conditions of the form

$$
\begin{gathered}
\alpha(x, t) u+\beta(x, t)(\partial u / \partial v(x, t))=g_{1}(x, t) \quad \text { on } S_{T} \text { or } S \\
u(x, 0)=g_{2}(x) \quad \text { on } B
\end{gathered}
$$

where $v(x, t)$ is a direction and

$$
\frac{\partial u}{\partial v(x, t)}=\lim _{P \rightarrow(x, t)} \frac{\partial u(P)}{\partial v(x, t)}, \quad P \in D .
$$

By a solution $u(x, t)=u\left(x_{1}, \cdots, x_{n}, t\right)$ of $L(u)=f(x, t)$ or $L(u) \geqq 0$ on $D$ or $D_{T}$, we mean a continuous function on $\operatorname{cl}(D)$ or $\operatorname{cl}\left(D_{T}\right)$ with $u_{x_{i} x_{j}}$ and $u_{t}$ continuous on $D$ or $D_{T}$.

We can now state our uniqueness theorem:

THEOREM 1. Let $u(x, t)$ be a solution of $L(u)=f(x, t)$ in $D_{T}$ subject to the boundary conditions (2), (3) with the added stipulation that $-v(x, t)$ points into $D_{T}, \alpha^{2}+\beta^{2} \neq 0$, and $\alpha, \beta \geqq 0$. Now suppose

(i) $c(x, t) \leqq 0$ and at each point $(x, t) \in S_{T}$, either $\alpha(x, t) \neq 0$ or $(x, t)$ and $v(x, t)$ satisfy the sphere property. Then if $v$ is another solution of $L(u)=f(x, t)$ subject to the same boundary conditions, $u=v$. The same condition is also true if instead of (i) we have

(ii) $c(x, t)$ is uniformly bounded for $t<T, \nu_{n+1}(t, x) \geqq 0$ for $(x, t)$ $\in S_{T} \cap\{0<t<T\}$, and for $(x, t) \in S_{T} \cap\{0<t<T\}$ one of the following holds:

(a) $(x, t)$ and $\nu(x, t)$ satisfy the sphere property,

(b) $\alpha(x, t)>0$,

(c) $\nu_{n+1}(x, t) \geqq 0$.

Proof. The key to the proof is the strong maximum principle for uniformly parabolic operators. The form we shall use is stated below 
and its proof follows from the methods and remarks given in $[2, \mathrm{pp}$. 163-177].

MAXIMUM PRINCIPLE. Let $u$ be a solution of $L(u) \geqq 0$ where $L(u)$ is defined as above and $c(x, t) \leqq 0$. Then if the maximum of $u$ on $\operatorname{cl}\left(D_{T}\right)$ occurs at $P \in D_{T}$, we have that $u(P)=u(Q)$ for any $Q \in D_{T}$ that can be connected to $P$ by a curve with nondecreasing $t$-coordinate.

The second result we need for the proof is the following directional derivative property. It can be found in $[1$, p. 49], or [2, p. 174].

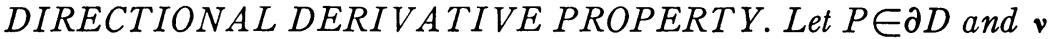
satisfy the sphere property with respect to the ball $K$. Furthermore, let $u$ be a solution of $L(u) \geqq 0$ where $L(u)$ is as above and where $u$ does not attain its maximum in int $(K)$. Then $(\partial u / \partial v)(P)>0$. Suppose $v$ is another solution of $L(u)=f(x, t)$ satisfying the same boundary conditions as $u$. Then $w=u-v$ is a solution of $L(w)=0$ satisfying the homogeneous boundary conditions $w=0$ on $B$ and

$$
\alpha(x, t) w+\beta(x, t)(\partial w / \partial v(x, t))=0 \quad \text { on } S_{T} .
$$

Now consider the case where (i) holds. Then if the maximum $M$ of $w$ on $\operatorname{cl}\left(D_{T}\right)$ occurs at $P$ and if $P \in D_{T}$, by the maximum principle and our assumptions on $D_{T}, M \leqq 0$. On the other hand, if $P=(x, t) \in S_{T}$, the boundary condition at $P$ is

$$
\alpha(x, t) w+\beta(x, t)(\partial w / \partial v(x, t))=0
$$

with $\alpha(x, t)$ and $\beta(x, t)$ being nonnegative and not vanishing simultaneously. Since $P$ is a maximum, $\partial w /(\partial v(x, t)) \geqq 0$. Hence $\alpha(x, t)>0$ contradicts (4) if $M>0$. But if $\alpha(x, t)=0$, then the directional derivative property can be used to obtain $\partial w /(\partial v(x, t))>0$ which also contradicts (4) if $M>0$. Hence $M \leqq 0$. Similarly, by considering - w, the minimum is nonnegative. Thus $w=0$.

In the case where (ii) holds, we may argue as above to obtain $M \leqq 0$ if $P \in D_{T}$, provided that the maximum principle applies. This is the case for $z=e^{-\lambda t} w$ when $\lambda \geqq 0$ large enough to make the coefficient of $z$ in the equation $(L-\lambda)(z)=0$ nonpositive. But $z \leqq 0$ implies $w \leqq 0$, so $M \leqq 0$ if $z \leqq 0$ for $P \in S_{T}$. Furthermore if $\lambda \geqq 0$ is chosen large enough, we may suppose that $z$ does not have a positive maximum on $\operatorname{cl}(D) \cap\{t=T\}$. On $S_{T} \cap\{0<t<T\}$, the boundary condition for $z$ is

$$
\left[\alpha(x, t)+\lambda \beta(x, t) \nu_{n+1}(x, t)\right] z+\beta(x, t)(\partial z / \partial v(x, t))=0 .
$$

However, since $\left[\alpha(x, t)+\lambda \beta(x, t) \nu_{n+1}(x, t)\right]$ and $\beta(x, t)$ are both nonnegative and not simultaneously zero, the same type of argument as 
used under (i) implies $z \leqq 0$ on $S_{T}$. Hence $M \leqq 0$. Similarly by considering $-w$, the minimum is nonnegative. Thus $w=0$.

\section{BIBLIOGRAPHY}

1. A. Friedman, Partial differential equations of parabolic type, Prentice-Hall Englewood Cliffs, N. J., 1964.

2. M. Protter and H. Weinberger, Maximum principles in differential equations, Prentice-Hall, Englewood Cliffs, N. J., 1967.

University of California, Los Angeles

\section{AN ELEMENTARY DERIVATION OF KHINTCHINE'S ESTIMATE FOR LARGE DEVIATIONS}

\section{MARK PINSKY ${ }^{1}$}

1. Introduction. In classical proofs of the law of the iterated logarithm, the estimate

$$
P\left(S_{n} / \sqrt{ } n \geqq a_{n}\right)=\exp \left[-\left(a_{n}^{2} / 2\right)(1+o(1))\right] \quad(n \uparrow \infty)
$$

plays a key role (see [3,pp. 41-49]). Here $S_{n}$ is a sum of $n$ independent identically distributed random variables with mean zero and variance one; $\left\{a_{n}\right\}$ is a fixed numerical sequence with some growth property. The first direct proof [2] of inequalities of this type involved cumbersome estimates of bilateral Laplace transforms and was restricted to bounded random variables. More recently, proofs of (1.1) and related inequalities have been derived as a corollary to global inequalities of the Berry-Essen type:

$$
P\left(\frac{S_{n}}{\sqrt{ } n} \geqq a\right)=\int_{a}^{\infty} \frac{\exp \left(-t^{2} / 2\right)}{(2 \pi)^{1 / 2}} d t+O\left(n^{-1 / 2}\right) \quad(n \uparrow \infty)
$$

when the error is uniform in $a \in(-\infty, \infty)$. The key observation in these proofs is that for a suitable choice of $a=a_{n}$, the error term in (1.2) can be absorbed into the Gaussian term (see [1, pp. 212-219], and [4]).

The purpose of this note is to point out that the idea of absorbing the error can be applied to a (much more easily proved) smoothed version of (1.2) to yield (1.1). The proof is based on Trotter's method of operators [5], which is presented in the lemma below. The whole point is that while Trotter's method seems incapable of yielding

Received by the editors July 15, 1968.

1 Research supported in part under contract N0014-67-A-0112-0015 at Stanford University, Stanford, California. 